This is an Accepted Manuscript of an article published by Taylor \& Francis in Cataloging \& Classification Quarterly on September 13, 2017, available online: http://www.tandfonline.com/10.1080/01639374.2017.1356782

\title{
Failure Modes and Effects Analysis (FMEA) for Cataloging: an Application and Evaluation
}

\author{
Abstract \\ Failure Modes and Effects Analysis (FMEA) is a proactive assessment tool originally created for quality \\ assurance in manufacturing industries. FMEA involves the assignment of rankings for frequency, \\ severity, and detection of errors within a process. Catalogers at ProQuest undertook an innovative \\ project to use FMEA to evaluate MARC record production. This article provides an overview of FMEA for \\ process evaluation and summarizes an application for cataloging. It considers the tool's value for error- \\ proofing in MARC record creation and how FMEA might be applied more effectively in a variable \\ environment.
}

Keywords: FMEA, quality assurance, error-proofing, MARC, cataloging

\section{Introduction}

ProQuest produces MARC bibliographic records in one of its metadata teams to support its Research Collections products. These records are distributed without ongoing maintenance, so it is essential that records are error-free at the time of distribution to customers. An established step in the quality control process had been proof-reading created records by a second cataloger, a costly but effective step. As an endeavor to possibly remove the proofing component while still ensuring quality records, the team creating bibliographic records for these products undertook a review of the MARC record creation process using Failure Modes and Effects Analysis (FMEA). The purpose of the analysis was to identify steps in the process that result in record errors and to prioritize the need to improve those steps.

FMEA is a preventative risk management tool used to anticipate and identify process or design failures and their causes, and to identify ways to resolve issues related to those failures before they 
reach the customer. FMEA results can be used to prioritize efforts for process improvements and to reduce failures and associated risks. FMEA is about identifying causes and effects, attributable to various conditions. Through FMEA, users can anticipate and prevent problems, reduce costs, shorten product development or production times, and achieve safe and reliable products and processes. Simply put, FMEA answers questions such as: what can go wrong? how likely is an error to occur? and if an error does occur, what are the consequences of that error? There are versions of FMEA for both the process to create a product (PFMEA) and the product design (DFMEA), with DFMEA also applying to services (a variant of product).

The library profession has long assessed the cataloging end-product for its ability to serve user needs. These assessments have inspired the introduction of new MARC fields, changes to descriptive standards, and even a vision for leaving the MARC record data model altogether. This application of FMEA was not to address possible errors in the design of the product (a DFMEA), but rather to analyze the process that can result in a flawed version of the intended product (a PFMEA). This article presents a case study of the ProQuest team's PFMEA application and an evaluation of the effectiveness of utilizing PFMEA for the cataloging process.

\section{Literature Review}

This application of PFMEA, to identify, prioritize, and address process steps with failure risks, is novel to the library environment. Studies on quality assurance initiatives for processes, such as authority control in the cataloging process, have been documented extensively, but no published articles discuss a technique to identify, prioritize, and address process steps with failure risks, as is accomplished with PFMEA. The authors did, however, identify three published resources related to manufacturing process applications in libraries and cataloging process assessment techniques that are relevant to cataloging work. 
Hsieh, Chang and $\mathrm{Lu}^{1}$ presented a review of quality management tools and techniques for libraries and information services, with a section specific to "quality by process control." They note that improving the process to avoid errors is more efficient than inspections to detect and then fix them after the fact. They emphasize the need for ongoing process improvement and describe a cycle for planning, carrying out, studying the effect, and further improving the process. While some specific tools are mentioned for process improvement, they do not reference FMEA. The FMEA tool is included, however, in their "quality by design" section, a DFMEA application. The article exposes some manufacturing-based models and techniques for possible use in libraries, with the valuable note in the conclusion that a refocus for an application in a new sector may be necessary.

Hunt ${ }^{2}$ described specific process improvement tools and techniques for cataloging and other technical services processes, based on practical experience discussed at improvement workshops at the University of Warwick. Noting that "there is very little research that has been undertaken that applies to process improvement techniques to cataloging, ${ }^{3}$ he presents seven improvement tools and techniques developed in manufacturing that can be useful for discussions of process improvement for libraries. FMEA was not included.

Nelson's article "Using Six Sigma and Lean in the Library"4 presents Six Sigma and related Lean techniques and tools from the manufacturing and business disciplines that have been used in libraries. She summarizes studies on their application to improve process efficiency such as reducing the time for acquisitions teams to obtain overseas purchases and another to reduce the time required to process incoming materials. Nelson then discusses ways in which libraries might successfully incorporate the proven process improvement techniques from the business world. In that discussion, she describes FMEA as a "powerful tool" to expose likely failures and to address them by improving the process or detection methods. While she recommends the use of PFMEA, she does not reference any known applications. 


\section{Introduction to FMEA}

FMEA was first developed in the 1950s to study malfunctions of military systems. ${ }^{5}$ Since then, use of the tool has expanded to analyze designs and processes in aerospace and defense industries in the 1960s, automotive companies in the 1970 s, ${ }^{6}$ to an everyday Lean Six Sigma practitioner's tool ${ }^{7}$ today. FMEA builds on the assumption that a failure is possible, and the FMEA team must establish how and when the failure might occur and what the resulting impact would be. ${ }^{8}$

A FMEA team starts with a process chart that provides an overview of the complete production process. ${ }^{9}$ The chart is used to help identify quality characteristics to be discussed in the FMEA error matrix, where the bulk of the FMEA work is done. The error matrix is a table that lists all process steps and all anticipated ways in which the step can go wrong (failure modes) with corresponding ratings for severity, occurrence, and detection. The ratings for those three factors are traditionally on a scale of 1 to 10, as shown in Figure 1.

[Figure 1: FMEA Scales for Severity, Occurrence, and Detection]

The team begins the error matrix by listing potential errors that could occur during the current production process. Then the team brainstorms and lists potential effects of each failure in the next column, ${ }^{10}$ followed by a column for the corresponding Severity rating. Potential causes of the error are considered and listed in the next column followed by the corresponding Occurrence rating. Then current controls are listed followed by the corresponding Detection rating. ${ }^{11} \mathrm{~A}$ risk priority number (RPN) is calculated for each step by multiplying the three ratings together. ${ }^{12}$ This number is intended to indicate the seriousness of the quality issues. ${ }^{13}$ The higher the number, the greater the importance. 
Once the RPN is calculated, a final column in the error matrix is used to suggest corrective action to reduce the severity of the error, to reduce the frequency of occurrence, or improve the detection, with an overall goal of reducing the RPN. For the errors that are prioritized for further action, the corrective action steps are transferred to a control plan where the team lists the actions to implement, assigns responsibility for each action, and states appropriate checks on actions and potential future actions. ${ }^{14}$

\section{Case Study}

The ProQuest metadata team creates full, "I" level MARC records in a process similar to librarians cataloging in more traditional library settings, using OCLC Connexion and Library of Congress tools such as Cataloger's Desktop and Classification Web. The team approached the PFMEA process looking for ways to create MARC records with minimal errors and to increase speed to market. The investigation focused on records created for ebooks in a variety of Western European languages. At the time of this PFMEA project, records were created according to Anglo-American Cataloguing Rules, $2^{\text {nd }}$ Edition (AACR2) standards, with authorized access points when available in the NACO authority file. Catalogers did not contribute new NACO records but formulated the needed text strings for those fields according to NACO standards.

The project kicked off with a team meeting to discuss quality control vs. quality assurance, PFMEA, and the major task of completing an error matrix. In preparation for the meeting, the project coordinator consulted professional literature addressing errors in vendor records, to determine the greatest concerns of the customers. During the meeting, catalogers discussed MARC error categories, the PFMEA error matrix and other steps for the project. The team identified three main categories of errors to use for evaluating potential failures in the FMEA model: errors affecting access (access errors), errors preventing records from being loaded into an ILS or ProQuest databases (critical errors), and 
errors unlikely to affect access or loading (other errors). ${ }^{15} \mathrm{~A}$ second consideration for categorizing errors came from questions within the group regarding objective vs subjective "errors." The cataloging process was outlined by creation method (original or derived) and field type (fixed, variable, and constant data), which were added to the error matrix as consideration for categorizing errors.

To fill out the error matrix, the team first identified potential failure modes in any given MARC field and the potential effects of each error. The effects were assigned a rating for Severity. Ratings for severity were scored high or low based on the seriousness of the perceived impact on the customer, on a scale of 1 to 10. (See Figure 1). Errors like incorrect notation of chief source were rated lower than missing name authority fields, given a library patron is less likely to have difficulty finding a resource based on a 588 note than a missing name. Next, potential causes of the error were identified, and those causes were assigned a rating for Occurrence (how likely it was to occur). Lower ratings were assigned for errors that are less likely to occur and higher ratings for common errors. Then current controls were identified that might catch that error, and a corresponding Detection rating was assigned. For the detection category, lower numbers were assigned when a cataloger would know that an error occurred. Specifically, a rating of 1 was only used for errors that could always be detected through automation, while a rating of 2 was used for errors frequently caught through manual detection. For each error, the Severity, Occurrence, and Detection ratings were multiplied together to get a Risk Priority Number, used to determine the multiplicative effect of each error. Errors with the highest RPN were considered a priority for process improvement investigation.

\section{Results}

The team organized the identified failure modes into three major components of a MARC record: constant data, fixed fields, and variable fields. A second grouping was assigned based on the type of data contained in each field: local unit information, company information, codes, matching 
fields, title and publication information, access/authority data, and notes. Using these categories, the team found the majority of failure modes existed in variable fields. Figure 2 shows the number of possible errors identified for each field category during the project. The team found the highest number of potential errors in title and publication information, followed by access point fields for a close second in potential errors.

[Figure 2: Failure Mode Counts]

While the failure mode counts reflect opportunities for errors within each MARC field category, the RPNs indicate the assigned risk for errors that occur. This part of the FMEA process involved discussion among the team members of how the error might impact the end user of the MARC record, the potential ways in which an error could occur, and the ways in which the error might be detected if it should occur.

For example, an incorrect 050 field resulted in one of the mid-to-lower RPNs. The severity of the error was not critical because it would not prevent a record from loading. Because these were e-book records, the team determined that a call number error would not be as severe as for a book on a shelf, but the error could prevent the record from correctly appearing in a call number browse feature or impact collection evaluation based on classification ranges. Potential Causes for the error were recorded as the cataloger simply making a typographical error or being distracted, or the cataloger needing more training on using Classification Web or the Cutter table. For the rest of the Error Matrix fields, the team recorded "manual proofing" for Current Detection Controls and "documentation" as Current Prevention Controls, which received moderate ratings. For the Control Plan, the team suggested more training on call numbers and using the tool Classification Web, as an opportunity to reduce the Occurrence value going forward. 
An example that received a higher RPN is a missing 650 field. The potential error was not critical because it would not prevent a record from loading, but it would be an access error. In the Error Effects field, the team determined the error would prevent the item from appearing in a subject search result or subject browse. Potential Causes for the error were recorded as a cataloger's lack of subject familiarity or poor authority searching skills. The team recorded "manual proofing" for Current Detection Controls, "documentation" and "training" as Current Prevention Controls. For the Control Plan, the team suggested additional training available through continuing education programs in the profession.

The MARC fields that returned the highest RPNs were the $1 X X$ and $7 X X$ fields followed by other access point fields that might be incorrect or missing. The next highest grouping of errors included almost every possible title entry error and then publication fields. The team assumed the major errors in a title field were based on incorrect transcription from the chief source of information. Most of the comments related to errors in access point fields were based on browse and search indexes that use that metadata.

[Figure 3: Average RPNs for MARC Field Categories]

The top-rated error was the 111 meeting name main entry field, at an RPN of 324. Other top errors include 100/110 wrong form of name, missing series title and subject headings, incorrect and missing title field data, and incorrect diacritics. The lowest rated errors were missing \$e in the $1 \mathrm{XX}$ and 7XX fields and general 500 notes missing, both with a rating of 1 . Other low-rated errors included note field indicators and missing 511 fields for video records. At this point, the team had not switched from AACR2 to Resource Description and Access, (RDA), so the impact of included or missing RDA elements in 
an AACR2 record were unclear. Therefore, ratings were not applied in situations where a 33X field might be missing (for example), as the team did not know or understand the impact of those fields.

The team conducted extensive discussions of possible causes for errors, how errors were detected, how errors were prevented, and actions taken upon finding an error in the control plan. Some possible causes included oversight, misinterpretation of rules, language barriers, unintentional typos, or general confusion. Detection method results were largely manual proofing by another cataloger, looking at a record field by field and comparing it to the item being cataloged. Some errors were caught by the validation tools built into the cataloging utility. The team's method of prevention was largely through cataloging documentation for the product and the initial training a new cataloger goes through when first starting on the team. Other detection methods relied on tools within the cataloging utility to verify field contents. Access point field errors could often be detected and corrected by controlling name and subject headings. Actions for correcting errors were typically resolved by a proofer noting the error and the cataloger fixing it. The project coordinator asked several times why the proofer couldn't fix the errors. The reason the team found against it was due to errors that were considered subjective and that the cataloger would be more likely to reduce mistakes after receiving the error feedback from the proofer. Actions for correcting subjective errors were noted as "Proofer notes error, cataloger evaluates and decides if it should change."

\section{Discussion}

The final step of the error matrix was brainstorming ideas to prevent future errors. Suggestions offered during the error matrix discussion were copied to create the control plan. Many suggestions revolved around better training up front or review of standards for all catalogers. A few suggestions resulted in changes to the constant data used to begin creation of MARC records and researching new tools that might aid in the proofing process. As an end result, the team added information to constant 
data, such as an underscore to the 856 field, which triggered a validation fail if a cataloger forgets to add URL information. Catalogers set derive record settings to exclude 856 fields from used records. The catalogers searched for additional macros to use during cataloging, created new documents to be used as a guide for higher rated errors (foreign language information, titles with numbers and parts, and/or meeting main entry field creation), and updated existing documentation for best practices. The catalogers implemented use of a new quality control tool, to add some automation to the manual proofing process. The catalogers now go through annual group meetings to review and edit documentation, making alterations based on changing current standards and interpretations. In general, the catalogers opt for a continuous improvement approach to their process, including education and training opportunities and new tools and techniques for improving the quality and efficiency of the cataloging process.

\section{Evaluation of PFMEA for Cataloging}

The PFMEA team identified specific areas to address process improvement initiatives, based on this application of PFMEA for cataloging. Although the tool had the aforementioned beneficial outputs, some caveats and suggestions for improved applications for cataloging are worth noting. These can be grouped into categories related to making appropriate preparations before starting the project, considering the traditional application of RPNs vs. how RPNs can be applied outside of traditional manufacturing processes, and understanding the time involved in completing the FMEA process.

\section{Prepare for the project}

Achieving successful FMEA results begins with understanding the fundamentals, definitions and procedures of FMEA, selecting the right FMEA projects, and completing preparation steps. ${ }^{16} \mathrm{~A}$ key preparation step that the team identified for cataloging was defining an error. The project coordinator 
at ProQuest provided the team with a brief presentation of the differences between quality assurance and quality control and a general overview of creating the PFMEA error matrix, which the group then began to populate. The concept of errors, however, had not been well established, and as the team worked to identify the failure modes for the cataloging process, it became clear that the concept of an error was inconsistent. While some metadata entered in a MARC field is unquestionably wrong (e.g., an invalid indicator or a typographic error), sometimes it is the lack of metadata that was perceived as an error. For a clearly stated series title on the piece that was not included in the record, the team interpreted that as an error. But during manual proofing process, a reviewer might "correct" a record that lacks an appropriate and useful subject heading even if other valid subject headings had been assigned. This is something the catalogers later decided to categorize as a "subjective error" (based on cataloger judgement) and did not consider it a failure mode. In support of that concept, a study that audited completed MARC records in a catalog did not investigate note fields because they found that they contained too much variation, and different may not be incorrect. ${ }^{17}$ The definition of an error should be stipulated at the beginning of the PFMEA to render the extensive rating process as simple and consistent as possible.

The team occasionally lost focus on what the intended product was, and began speculating on possible failure modes for RDA records (as they were creating AACR2 records at that time), or what the severity of an error might mean for metadata mapping to a linked data model. Preparing for the PFMEA should involve a clear explanation of what an error-free product is, and having that posted in view of the team as a constant reminder while working through the error matrix would have helped the team to stay on task.

The members of the project team should represent a range of experience because different perspectives will result in different rating assignments. A small group of people who all do the same thing may not be aware of the things they don't know. ${ }^{18}$ This team was composed only of vendor 
catalogers, none of whom had a background at an institution that loaded vendor records into a local system. Although the team tried to imagine the severity of an error from the customer's perspective, they had no specific knowledge of how different catalog systems or record-loading processes might be impacted by particular errors.

\section{RPNs and what they really mean}

The concept of the RPN creation, the multiplication of the Severity, Occurrence, and Detection ratings, is not supported by evidence that such a calculation is the best way to compare the error risks.${ }^{19}$ As an example, a one point difference for one factor at the high end can result in an RPN difference of $100(10 * 10 * 10=1000$ vs. $10 * 10 * 9=900)$, while a one point difference for one factor at the low-end results in an RPN difference of only $1(1 * 1 * 1=1$ vs. $1 * 1 * 2=2)$. The two RPNs at the high end of the scale seem significantly different, while those at the low end seem about the same. To remove the biased perception from the multiplicative nature of the RPN, the results could be assigned a corresponding ordinal number, which for a 10-point scale would result in the high-end example having RPNs of 119 and 120 and the low-end example having RPNs of 1 and 2, showing that their difference is the same. ${ }^{20}$

The Risk Priority Number is not a consistent measure of real risk, rather a rating of how the team considers the risk, ${ }^{21}$ and to account for this another proposed revision of the RPN calculations involves weighted sums. ${ }^{22}$ That calculation allows different team members to assign different values, which the developers of the weighted sums calculation believe are better suited for an uncertain environment. If different perspectives mean different value assignment, then the resulting value should reflect those differences rather than requiring one that everyone agrees on. ${ }^{23}$ The rethinking of RPN calculations can involve complex mathematical formulas, but what is of particular interest is how users have been able to adapt the RPN to allow FMEA to better suit different scenarios and desired outcomes. 
For cataloging, the ratings for Occurrence are also based on impressions rather than solid data. For this team, the Occurrence ratings came primarily from experience in creating MARC records and proofing each other's records. Relying on error occurrences that are generated from database error reports might seem more appropriate, but there are errors that could not be detected by machine generated data.

After completing a PFMEA that used the traditional RPN calculation, the catalogers questioned whether the three factors should be considered equally. Following the traditional RPN, the three factors are equally weighted, and different combinations of values for Severity, Occurrence and Detection would result in the same RPN. As the team reviewed the RPNs of the project, it became clear that this is too simplified a perspective, and that not all errors with the same RPN are truly equal. Though the group did not create an actual weighting factor, they felt that the errors with high Severity value should be given greater attention in the control plan, as illustrated with Figure 4.

Figure 4 shows the RPNs for errors that rate at the extreme ends for Occurrence, Detection, and Severity when applying a 10-point scale. The corner labeled A correlates to an error with high Severity but low Occurrence and Detection. This scenario could be interpreted as "errors that don't happen often, are usually detected, but have a serious impact when not caught." The region near the corner labeled B could be interpreted as "errors that happen a lot and are rarely detected, but with no significant impact." The RPN for an error at A would be 10 (ordinal value of 10) and at B would be 100 (ordinal value of 46 ).

[Figure 4: the RPN cube for a 10-point scale]

The team concluded that a failure mode that involved a high Severity value should be addressed even if the calculated RPN was not high relative to other failure modes. They felt that the point at which 
Detection happened could be important in failure mode prioritization. There are economic

consequences related to the amount of time an error goes undetected and how much effort is required to then correct the error. Detection at the time an error is made is more efficient and should result in a lower Detection value. Errors that could only be detected by manual proofing should be prioritized for improvement over those that are detected by automated validation tools.

\section{It can take a long time}

Many who have applied the PFMEA tool have noted that it is time consuming, and possibly frustrating, even for a production process using standard parts that are assembled or enhanced in a regular, predictable way. ${ }^{24}$ Creating MARC records involves many variables in the production parts, including the characteristics of the item to be cataloged and possible errors already in place when deriving from an existing record. There are many MARC field creation steps that had to be considered, and creating the error matrix was very involved for each of these steps. In the end, the team spent a great deal of effort on areas that turned out to be of little concern.

However, if the time and effort is not spent on the error matrix and the FMEA is not detailed enough, errors can be missed, making the process ineffective. This lack of detail would result in areas of concern with little to no definition to the problem. ${ }^{25}$ If a team spends too little time brainstorming, solutions may be lacking and high risk problems could remain unresolved.

This PFMEA project was designed to address every possible error in every MARC field, and the time to complete the project was much lengthier than expected. After many hours of work over several weeks, the team became less engaged in discussing and assigning values. One possible way to reduce the time needed for this process is to assign one of three rates, for Low, Medium, and High. There is evidence that reducing the rating options will minimize meaningless debate on close values ${ }^{26}$ and will not greatly impact the results. ${ }^{27}$ This simplified 3-point rating would quickly identify the high Severity 
errors, which had become a priority for this application. Then the subset of highly rated errors could be further refined with a larger scale if necessary.

\section{Conclusions}

The cataloging team's key takeaway from the PFMEA process was recognizing the need to ensure that local practices comply with external standards and support evolving metadata use. Even though additional quality management tools were added to cataloging practices, the ongoing work of reviewing points made in the control plan has kept the team in the practice of continuous improvement. Due to ongoing changes in metadata standards and the introduction of new applications of that metadata, continuous improvement is necessary for cataloging processes. Hsieh, Chang, and Lu, in their discussion of manufacturing-based models and techniques for managing quality in library and information services, conclude that a framework of quality management must be established for the library by promoting a quality culture. ${ }^{28}$ The catalogers saw value in having ongoing quality management conversations that are not limited to managers. The FMEA team approach is useful to communicate the importance of quality production and to brainstorm improvement plans using the experience and expertise of the people doing the work.

The FMEA approach also gave the catalogers a different way of thinking of their processes. Using additional quality management tools provides catalogers with added opportunities for error detection, which should ideally happen at the time the mistake is made. Having to go back to the record as well as needing to inspect the item that was already cataloged is much less efficient. The team's suggested solution to catching errors at the time of production requires keeping basic understanding of rules, practices, and standards at a high level. Participating in industry webinars and monitoring professional listservs are useful for ongoing education as standards continuously evolve and new 
cataloging tools are created. Improving means of automated detection should always be considered, because human proofing has its own risk of failure in addition to being less efficient. ${ }^{29}$

While the application of PFMEA can result in process changes that directly improve the occurrence or detection of errors, it is less likely to impact the severity of errors. ${ }^{30}$ Changes to local cataloging processes will not change the impact of the errors, but catalogers can work to influence the development of systems that make use of cataloging metadata today or in the future. For example, reconciling MARC data for conversion to a linked data model will be impacted by errors and missing data, and the severity of those will depend on the conversion tools. The cataloging process would benefit from repeated FMEA-like analysis and related process changes, with the understanding that the original intent of FMEA was for a production environment where the end product is intended to be exactly the same every time. For industries like automotive production, that have experienced extensive FMEA work over a quarter century, the "critical few" may have already been addressed, leaving a "trivial many" that are hard to prioritize or even address. ${ }^{31}$ However, this is not the case for cataloging where critical errors in the process are still very possible and need to be addressed. If catalogers think beyond MARC records, larger questions and potential problems arise. It can be said, in future environments, continuous improvement tools such as PFMEA will serve an ongoing need for quality metadata for libraries.

\section{Endnotes}

1. Pao-Nuan Hsieh, Pao-Long Chang, and Kuen-Horng Lu, “Quality Management Approaches in Libraries and Information Services," Libri 50 (2000), 191-201.

2. Stuart Hunt, "Improving Performance in Cataloguing and Technical Services Workflows," Catalogue \& Index 161 (Dec. 2010), 10-15.

3. Ibid, 10. 
4. Elizabeth Nelson, "Using Six Sigma and Lean in the Library," College \& Undergraduate Libraries, 22, no. 3-4 (2015), 312-324. DOI: 10.1080/10691316.2015.1070701.

5. TM Kubiak, "Conducting FMEAs for Results." Quality Progress 47, no. 6 (Jun 2014), 42.

6. Steven Kmenta, “Advanced Failure Modes and Effects Analysis: a Method for Predicting and Evaluating Failures in Products and Processes" (PhD diss., Stanford University, 2001), 3.

7. Kubiak, "Conducting FMEAs for Results," 42.

8. Janet Webber, "FMEA: Quality Assurance Methodology," Industrial Management + Data Systems 90, no. 7 (1990), 21.

9. Lian-yu Zheng, Kwai-sang Chin, and Li Wei, "Knowledge-Enriched Process FMEA Model for Process Planning," Asian Journal on Quality 3, no. 1 (2000), 15.

10. William Goble, "The FMEA Method: a Powerful Reliability Tool for Data Analysis That Lasts for Decades," Intech March/April (2012), 17.

11. Webber, "FMEA," 21.

12. Goble, "FMEA Method," 17.

13. Zheng, "Knowledge-Enriched Process FMEA," 18.

14. Webber, "FMEA," 22.

15. Stacie Traill, "Quality Issues in Vendor-Provided E-Monograph Records." Library Resources \& Technical Services 57, no. 4 (October 2013), 216.

16. Carl S. Carlson, "Which FMEA Mistakes Are You Making?," Quality Progress 47, no. 9 (2014), 37.

17. Ann Chapman and Owen Massey, "A Catalogue Quality Audit Tool," Library Management 23, no. 6/7 (2002), 320.

18. Carlson, "Which FMEA Mistakes," 39. 
19. Yuxian Du, Xi Lu, Xiaoyan Su, Yong Hu, and Yong Deng, "New Failure Mode and Effect Analysis: An Evidential Downscaling Method," Quality and Reliability Engineering International 32 (2016), 738.

20. Donald J. Wheeler, "Problems with Risk Priority Numbers." Quality Digest Magazine (June 27, 2011). Accessed February 12, 2016. http://www.qualitydigest.com/inside/quality-insiderarticle/problems-risk-priority-numbers.html.

21. Kmenta, "Advanced Failure Modes and Effects Analysis," 9.

22. Du et al., "New Failure Mode and Effect Analysis," 740-741.

23. Ibid., 745.

24. John J. Casey, Strategic Error-Proofing: Achieving Success Every Time with Smarter FMEAs (New York: CRC Press, 2009), 7.

25. Carlson, "Which FMEA Mistakes," 40.

26. Kubiak, "Conducting FMEA for Results," 44.

27. John Dawes, "Do Data Characteristics Change According to the Number of Scale Points Used?," International Journal of Market Research, 50, no. 1 (2008), 75.

28. Hsieh, Chang, and Lu, “Quality Management Approaches," 200.

29. Casey, Strategic Error-Proofing, 8.

30. Ibid., 5 .

31. Ibid., 6. 
Figure 1.

FMEA Scales for Severity, Occurrence, and Detection

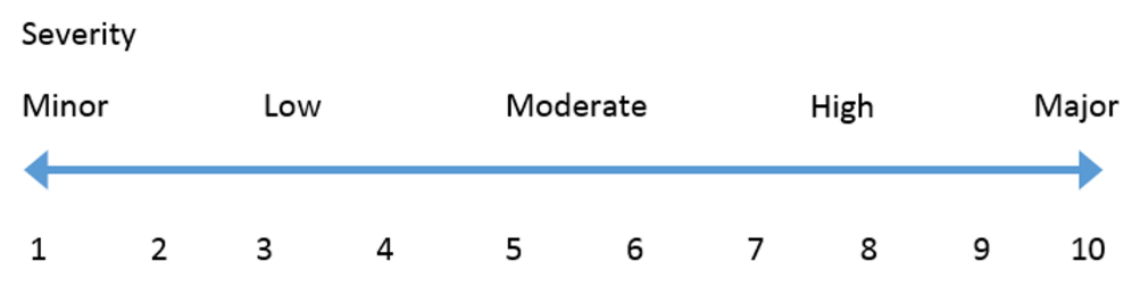

Occurrence

$\begin{array}{llllllllll}\text { Unlikely } & \text { Isolated } & & \text { Occasional } & & \text { Frequent } & & \text { Inevitable } \\ 1 & 2 & 3 & 4 & 5 & 6 & 7 & 8 & 9 & 10\end{array}$

Detection

\begin{tabular}{lllllllllll} 
Always & & Often & & Occasional & & \multicolumn{2}{c}{ Seldom } & & Cannot \\
\hline & 2 & 3 & 4 & 5 & 6 & 7 & 8 & 9 & 10
\end{tabular}


Figure 2.

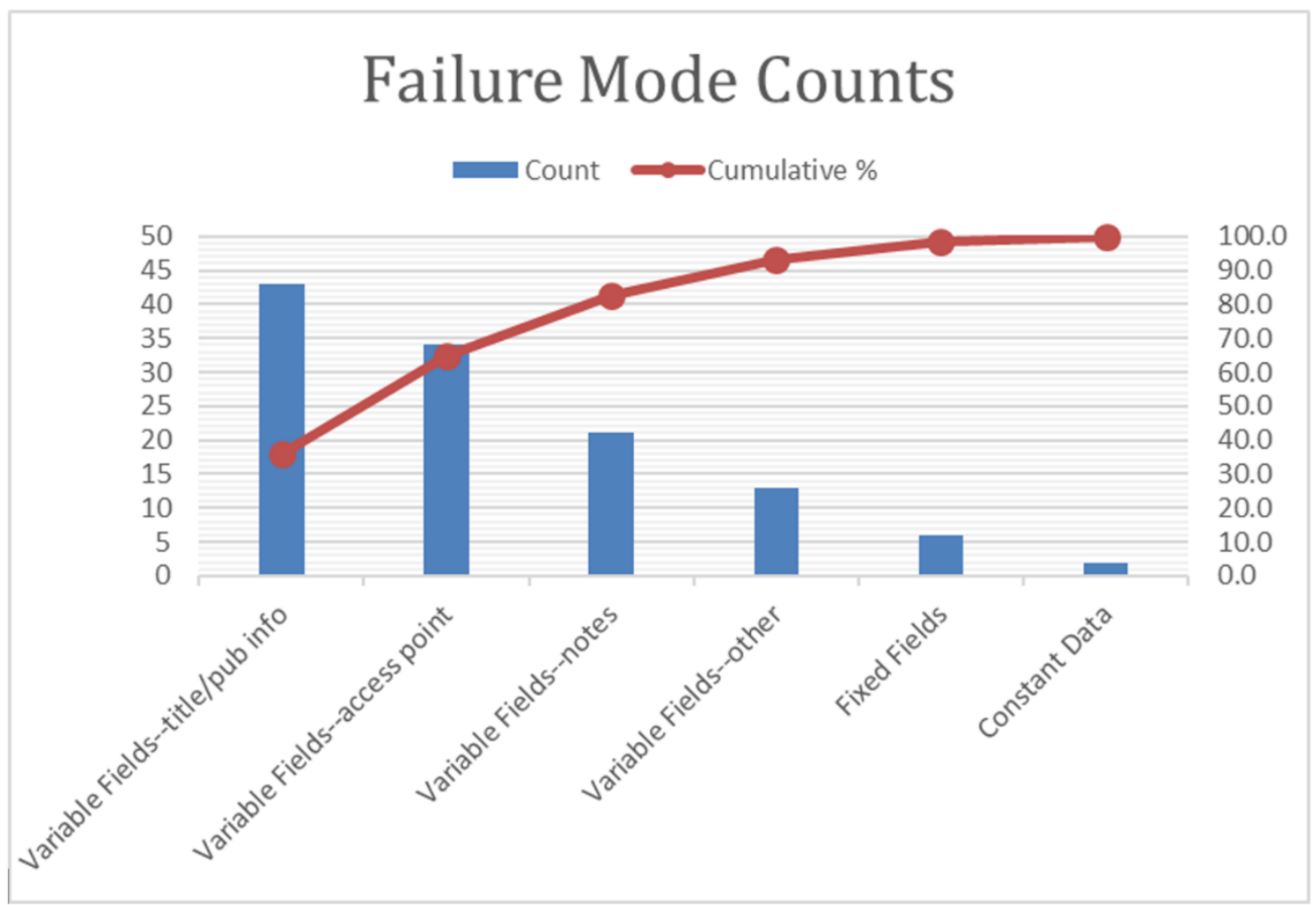


Figure 3.

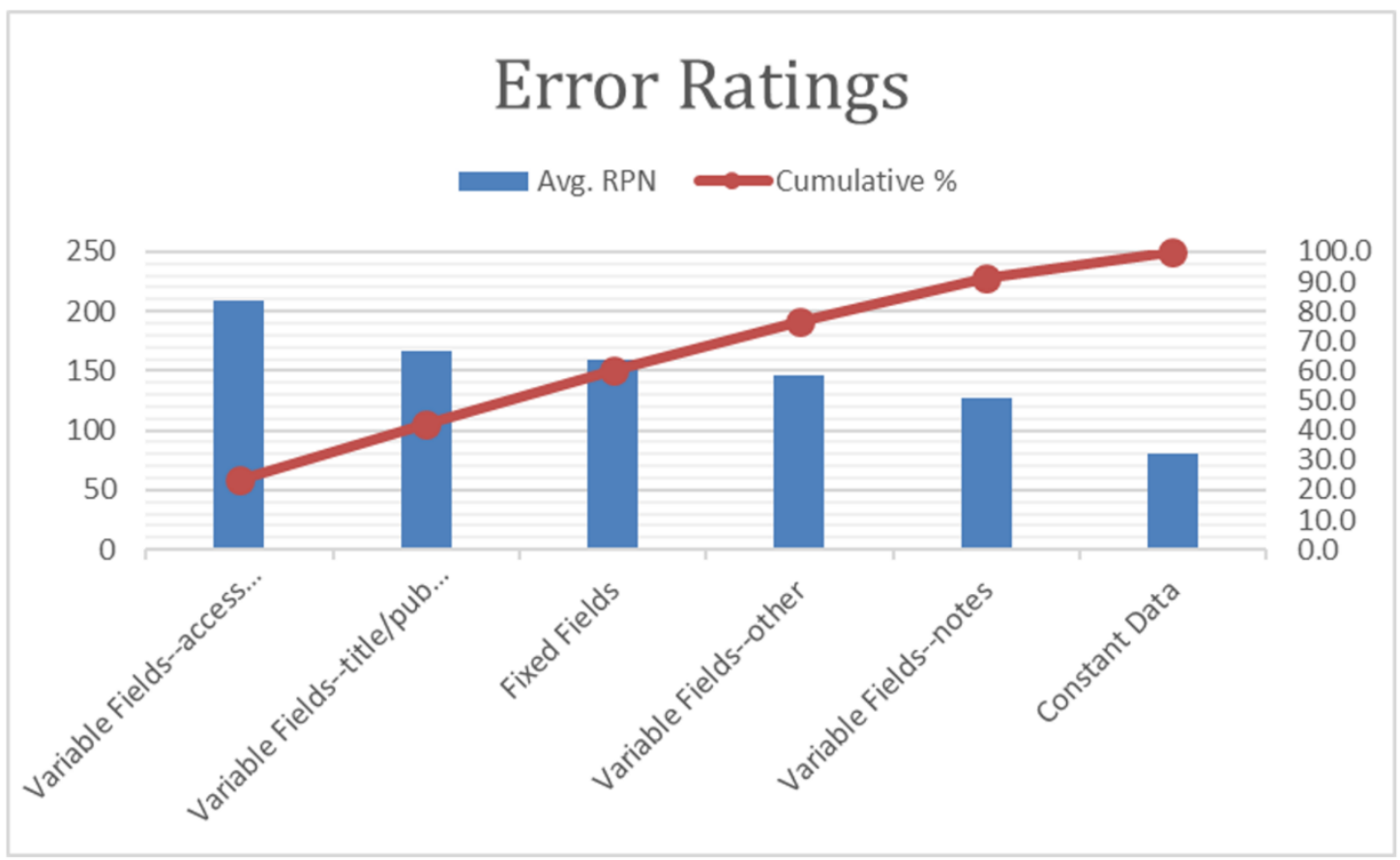


Figure 4.

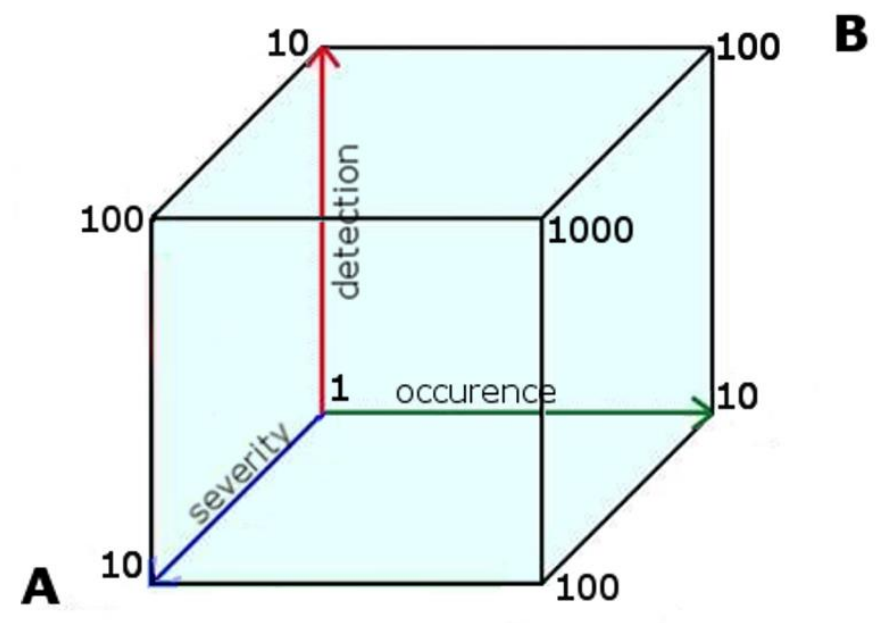

